This item was submitted to Loughborough's Research Repository by the author.

Items in Figshare are protected by copyright, with all rights reserved, unless otherwise indicated.

\title{
A novel yet effective motion artefact reduction method for continuous physiological monitoring
}

PLEASE CITE THE PUBLISHED VERSION

http://dx.doi.org/10.1117/12.2044640

\section{PUBLISHER}

(C) Society of Photo-Optical Instrumentation Engineers

\section{VERSION}

VoR (Version of Record)

\section{PUBLISHER STATEMENT}

This work is made available according to the conditions of the Creative Commons Attribution-NonCommercialNoDerivatives 4.0 International (CC BY-NC-ND 4.0) licence. Full details of this licence are available at: https://creativecommons.org/licenses/by-nc-nd/4.0/

\section{LICENCE}

CC BY-NC-ND 4.0

\section{REPOSITORY RECORD}

Alzahrani, Abdullah, Sijung Hu, Vicente Azorin-Peris, Roy S. Kalawsky, Xiaolong Zhang, and Changqing Liu. 2019. "A Novel yet Effective Motion Artefact Reduction Method for Continuous Physiological Monitoring". figshare. https://hdl.handle.net/2134/21299. 


\title{
A novel yet effective motion artefact reduction method for continuous physiological monitoring
}

\author{
A Alzahrani ${ }^{\mathrm{a}}, \mathrm{S} \mathrm{Hu}^{\mathrm{a}}{ }^{*}, \mathrm{~V}$ Azorin-Peris ${ }^{\mathrm{a}}$, R Kalawsky ${ }^{\mathrm{a}}, \mathrm{X}$ Zhang $^{\mathrm{b}}, \mathrm{C}$ Liu $^{\mathrm{b}}$ \\ ${ }^{a}$ School of Electronic, Electrical and Systems Engineering, ${ }^{b}$ Wolfson School of Mechanical and \\ Manufacturing Engineering Loughborough University, \\ Ashby Road, Loughborough, Leicestershire LE11 3TU UK
}

\begin{abstract}
This study presents a non-invasive and wearable optical technique to continuously monitor vital human signs as required for personal healthcare in today's increasing ageing population. The study has researched an effective way to capture human critical physiological parameters, i.e., oxygen saturation (SaO2\%), heart rate, respiration rate, body temperature, heart rate variability by a closely coupled wearable opto-electronic patch sensor (OEPS) together with real-time and secure wireless communication functionalities. The work presents the first step of this research; an automatic noise cancellation method using a 3-axes MEMS accelerometer to recover signals corrupted by body movement which is one of the biggest sources of motion artefacts. The effects of these motion artefacts have been reduced by an enhanced electronic design and development of self-cancellation of noise and stability of the sensor. The signals from the acceleration and the opto-electronic sensor are highly correlated thus leading to the desired pulse waveform with rich bioinformatics signals to be retrieved with reduced motion artefacts. The preliminary results from the bench tests and the laboratory setup demonstrate that the goal of the high performance wearable opto-electronics is viable and feasible.
\end{abstract}

Keywords: Accelerometer; Artefact motion; real-time physiological monitoring and assessment, opto-electronic sensor, personal healthcare.

\section{INTRODUCTION}

With the demand for increased personal healthcare for today's growing and ageing population, Chronic diseases, i.e., cardiovascular disease $(C V D)$, stroke and diabetics are the main causes of disability and death ${ }^{[1]}$ require cost-effective healthcare monitoring to observe health conditions continuously in order to prevent chronic conditions from deteriorating. Telemedicine technology is an important means of non-invasive healthcare monitoring which is well developed and is becoming a valuable means to reducing the costs of treatment, increasing service quality in the health care system and save time. As indicated by WHO ${ }^{[2]}$, continuous monitoring is one of effective means to not only assess physiological status that are related to these diseases, but also provide indications of disease progression and make it easier to manage daily based medicine and therapy. It is vital to take such a cost-effective means to minimize the consequences of these diseases.

Opto-physiological modelling (OPM) based Photoplethysmography (PPG) ${ }^{[3]}$, is an important non-invasive optical technique that is used to measure dynamic change of tissue optical properties with blood volume change in the microvascular bed of tissue. The principle of detection depends on Lambert Beer's law which predicts the intensity of reflected/refracted light from red and IR to the pulsate area in the body such as a finger, forehead or wrist and detects the transmitting or reflecting back light by the detector. The effective vital human signs monitoring and assessment are based upon the better understanding of opto-physiological interaction of illumination with biological tissue and is one of the most important ways to offer continuous yet accurate health assessment technique. As expressed ${ }^{[4]}$, the physiological parameters, i.e., oxygen saturation $\left(\mathrm{SaO}_{2} \%\right)$, heart rate, respiration rate even heart rate variability (HRV) are strongly related to physical activities that can be tracked by a 3-axes MEMS accelerometer. Meanwhile, the detection of physical activity can be used to recover signals corrupted by body movement which is one of the biggest sources of motion artefacts. These motion artefacts can be reduced by an appropriate electronic design and development of algorithms for self-cancellation of noise and stability improvement of the sensor. Referring to available principle of the opto-

`.Hu@lboro.ac.uk; phone +44 1509227059

Design and Quality for Biomedical Technologies VII, edited by Ramesh Raghavachari, Rongguang Liang, Proc. of SPIE Vol. 8936, 89360G · C 2014 SPIE · CCC code: 1605-7422/14/\$18 · doi: 10.1117/12.2044640 
physiological properties and oxygen saturation measurement in the blood ${ }^{[3,5]}$, the absorption of light differs, as usually deoxygenated haemoglobin in near infrared (IR: $910 \mathrm{~nm}$ ), and oxygenated transmission through near red (RED: $660 \mathrm{~nm}$ ). According to whether haemoglobin is saturated by oxygen or not ${ }^{[6]}$, the level of blood oxygen saturation can be determined as indicated in Figure 1.

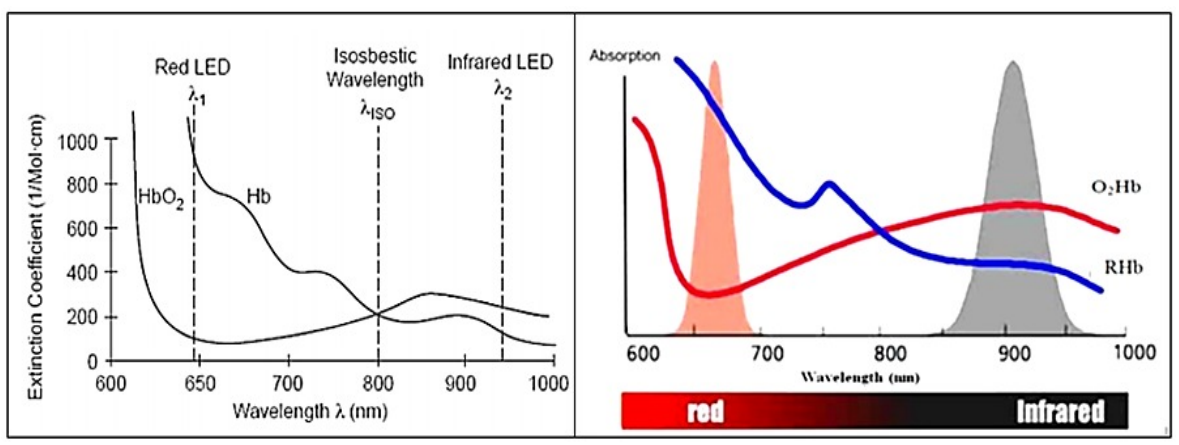

Figure 1. The wavelength regions of the red LED and the infrared IR illumination in pulse oximetry ${ }^{[6]}$

To this end, Coyle $\mathrm{S}$ et $a l^{[7]}$ have researched textile-based wearable sensors to assess physiological signals and body kinematics during exercise. Curone D et al. ${ }^{[8]}$, developed smart garments (ProeTEX) for vital health state monitoring in emergency operators. A woven textile to integrate photodiodes and light emitting diodes has been created to enable nearinfrared spectroscopy (NIRS) systems to monitor arterial oxygen saturation and oxygenated and deoxygenated hemoglobin in human tissue ${ }^{[9]}$. Although a good progress of wearable sensors and actuators has been made for human vital signs monitoring and assessment, there are still few challenges ${ }^{[10]}$ remaining in either clinical setup or continuously personal healthcare with the realizable and reliable technologies.

Our research has developed an effective way to capture critical physiological parameters through a closely coupled wearable OEPS together with a real-time wireless communication capability. The detailed objectives were: 1) better understanding of interaction of illumination to biological tissue in order to provide continuous yet accurate health assessment; 2) physical activity monitoring via acceleration detection to be used for recovering signals corrupted by body movement; and 3) an appropriate electronic design and development of algorithms for self-cancellation of noise and increased stability of the opto-electronics patch sensor (OEPS).

\section{METHOD}

\subsection{SYSTEM DESIGN FOR WEARABLE VITAL HUMAN SIGNS MONITORING}

A simple block diagram as shown in Figure2 illustrates the system design of the continuous physiological monitoring including opto-electronic patch sensor, a 3-axis accelerometer and a body temperature sensor.

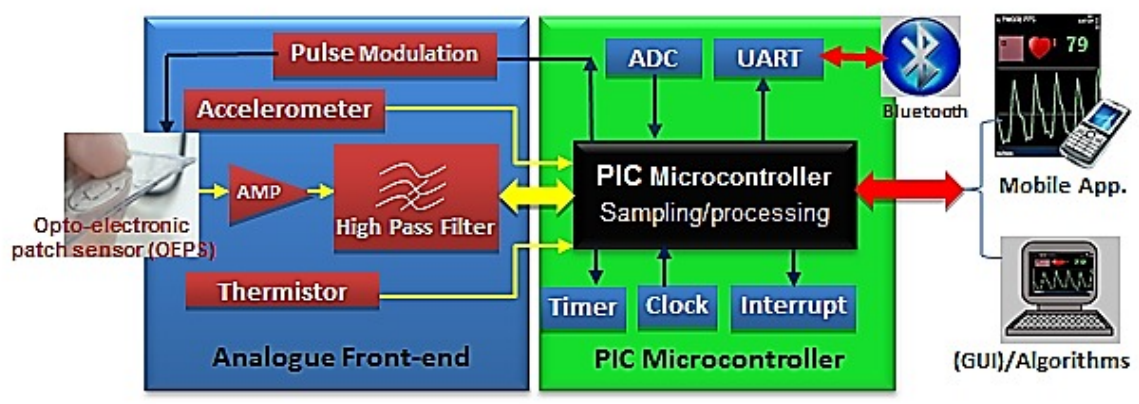

Figure 2. The system design of reflectance OEPS for continuous physiological monitoring.

A motion reduction physiological monitoring system comprises of three aspects: 1) Analogue Front-end to catch the pulsated waveform signals via a wearable opto-electronic sensor (customized) associated a pre-amplifier and a high pass filter, to detect motion artefact using a 3axis accelerometer (ADXL337, Analogue Device Co.) and a body temperature 
sensor (SMD3 0603, Measurement Specialties); 2) PIC Microcontroller to implement a low pass filter, to sample the signals $(A D C)$ and to process the captured signals then make data ready to be transferred to a smartphone via a Bluetooth module using a UART protocol; and 3) Mobile Communication unit to receive the data then process the desired signals in further, and finally display them in the form of graphic and reading numbers.

\subsection{OPTO-PHYSIOLOGICAL MONITORING ENGINEERING MODEL WITH A MOTION REDUCTION METHOD}

In order to understand how this technique works and the underlying assumption of the system, a series of implementations and algorithms were derivative.

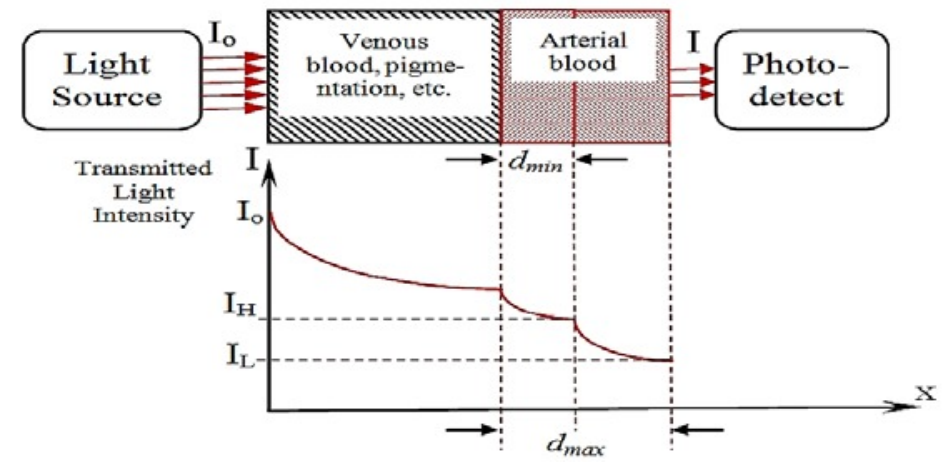

Figure 3. Lambert-Beer's law in pulse oximetry.

Figure 3 shows that the light intensity $I_{0}$ passes through different layers and detected as transmitted or reflected light $I$. During the systolic phase of the heart, the blood is distributed in the body through blood vessels; and therefore, the diameter of the arterial vessels increases and contains more blood and that can be expressed as $d_{\max }$. Thus in this stage, extra light will be absorbed, which will result in less light being detected by the photo detector as $I_{L}$. As more blood enters the vessels, less light is recorded in the detector. In contrast, during heart diastolic mode, the opposite processes will occur; blood volume in the vessel is reduced $d_{\min }$ and thus, less light will be absorbed, which then results in greater intensity detection and this represent as $I_{H}$. As the light rises and falls in the photo detector, the fluctuating waveform signal (AC component, $P P G)^{[1]}$ appears due to the changing diameter of blood vessel (pulsation) as shown in Figure 4. However, in the layers where the non-pulsatile tissues are presented, the constant absorbance will appear as a $D C$ component and thus can be removed by using an appropriate method. The $P P G$ provides critical health information, such as heart rate, heart rate variability, blood pressure and oxygen saturation ${ }^{[12]}$.

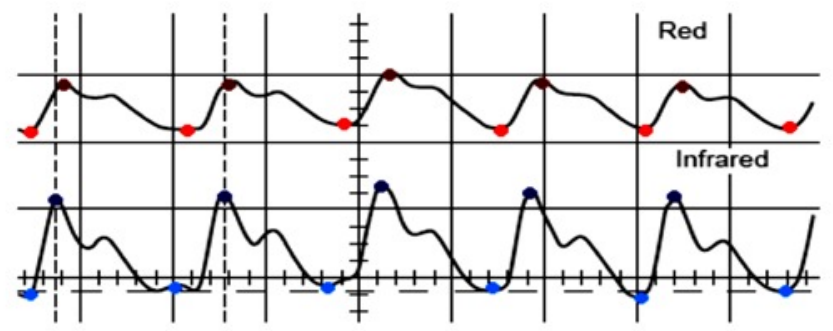

Figure 4. The $P P G$ waveform Red $L E D$ and Infrared $I R$ due to arterial pulse ${ }^{[13]}$

However; the $P P G$ signal is susceptible to artefact noises that originate from different sources, such as ambient light and motion artefacts caused by voluntary or involuntary movement. Figure 2 illustrates an automatic motion artefact cancellation technique. The technique behind this method depends on the fact that the system design uses the corrupted $P P G$ signal as a primary input and the motion signal as a reference input, which is correlated with the noise in the primary input. The two correlated signals cancel each other out and an uncorrelated signal thus is a reduce noise PPG signal, remains. Both signals resulting from the accelerometer and the sensor were digitized in the microcontroller in 
order to perform further processing. In the absence of movement, no signal comes out from the accelerometer, and only one channel of $A D C$ of the detection sensor is executed.

The motion artefacts in opto-physiological monitoring are mainly due to modification of the optical properties of the internal tissues and due to external or outer sources such as electromagnetic, electronic, power supply, pressure and ambient light. These kinds of modification affect the Beer-Lambert equation and therefore can be interpreted as in equation 1.

$$
I(t)=I_{0}(t) \cdot \eta_{\text {static }} \cdot \eta_{\text {pulse }}(t) \cdot \eta_{\text {motion\&gravity }}(t) \cdot \eta_{\text {biological tissues }}(t)
$$

Where $\eta_{\text {static }}$ is the static attenuation due to the constant tissues (non-pulse, bones..), $\eta_{\text {pulse }}(t)$ is the artefact that occurs along with pulsatile absorption, $\eta_{\text {motion\&gravity }}(t)$ is due to change of position and physical movement and $\eta_{\text {biological tissues }}(t)$ is due to the dynamic internal change of tissues and psychological effect.

In the absence of physical movement, the relaxing mode only both contribution of $\eta_{\text {static }}$ and $\eta_{\text {pulse }}(t)$ is presented. The static contribution is the artefact or undesirable constant value that results from non- pulsatile tissues which absorbs the same amount of the light during the time changing. This static contribution appears as a $D C$ component and can be filtered out by using a proper method such as high pass filter with cut-off frequency close to zero. Therefore, in the 'rest' measurement mode where there is no physical action the remaining contribution artefact is the pulse $\eta_{\text {pulse }}(t)$ this can be expressed as the following.

$$
\eta_{\text {pulse }}=\eta_{\text {ambient }}(t) \cdot \eta_{\text {electromagnatic }}(t) \cdot \eta_{\text {electronic \& power supply }}(t) \cdot \eta_{\text {coupling alignment }}
$$

Regarding the electromagnetic $\eta_{\text {electromagnatic }}(t)$ and power supply $\eta_{\text {electronic \& power supply }}$ noises, they appear as considerable higher frequency artefact that can be removed by designing a proper low pass filter. The ambient light artefact $\eta_{\text {ambient }}(t)$ could be isolated from the actual desired signal by using a lightproof cover to prevent the optical sensor being exposed to the ambient/external light or, multiplexing techniques that use subtractive method. The coupling artefact $\eta_{\text {coupling alignment }}$ is due to the mismatch alignment between the source of the light and the detector and this cause a direct optical path to the detector without passing through the tissues and corrupt the PPG signal. Usually, this occur in the transmission mode design and could be avoided be using a proper design and a ligament the source and the detector in a proper way.

As a consequence, when the measurement has been taken in relaxing ('rest') mode or in the absence of body movement, it is straightforward to recover the pulsatile signal or dynamic waveform with the consideration of the above procedures. However, when the subject or body is moving, the signal is modified as a result of the motion and gravity $\eta_{\text {motion\&gravity }}(t)$ as well as the dynamic internal change of the tissues $\eta_{\text {biological tissues }}(t)$. These signal variations are varying with the time; therefore, the desired $P P G$ signal is corrupted. However, a study ${ }^{[14]}$ however indicates that the greatest and worst contribution artefact noise is produced from physical activity and body movement $\eta_{\text {motion\&gravity }}(t)$ . In addition, the contribution artefact caused by biological alignment tissue $\eta_{\text {biological tissues }}(t)$ is further small compare to the contribution caused by of the motion artefact. Therefore, a novel technique has to be applied in order to cancel the contribution of the motion artefact and to acquire an accurate estimation of the desire PPG signal. In the proposed approach, an analogue tri-accelerometer which can detect acceleration and movement in all three axes in respect of gravitational acceleration and provides a reliable motion reference.

To achieve efficient removal of the contribution of the motion artefact, an automatic differential measurement by means of a normalization approach is used. This approach uses a tri-accelerometer to determine the physical movement along three axes resulting in more accuracy since the three vector $x, y$ and $z$ will be present. Therefore, the total acceleration vector will be presented as a combination vectors of $x, y$ and $z$. 


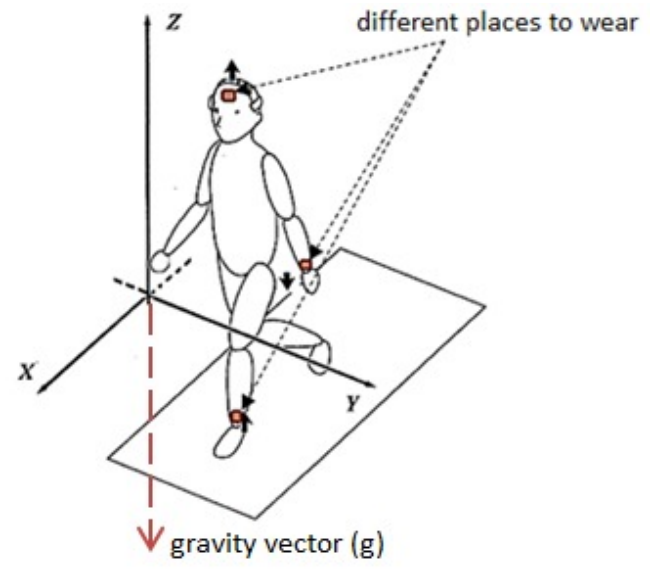

Figure 5. Accelerometer coordinate system in relation to the gravity vector and the walking direction.

Figure 5 shows that the OEPS can be adhered in different position on the body which can detect the PPG signal during walking. However, the contribution of motion artefact will be different dependent on the direction or movement. In other words, if the OEPS is placed in the wrist, it will generate different motion artefact than if it is adhered to the forehead. In the present design when movement takes place, the accelerometer produces a signal proportional to the body movement and regardless of the direction of the movement and the position of the OEPS, since the system design uses a combination circuit. Therefore, the vector $R$ is the force vector that represents the movement and can be measured through the projection of combination of $R x, R y$ and $R z$. Therefore, accelerometer output (acceleration) is measured in the three dimensions ( $X, Y$ and $Z$ vectors), and then integrated to represent movement over a certain time using the square root of the sum of squares for each individual vector. The integrated signal for movement over time is represented by vector magnitude units (VMU) ${ }^{[15]}$ which is called acceleration synthesization.

$$
V M U(R)=\sqrt{R x^{2}+R y^{2}+R z^{2}}
$$

The values of projection that appear in the corrupted $P P G$ are related to the values produced from the accelerometer. The output is analogue which can be converted into a voltage level through the $A D C$ of the microcontroller. However, the magnitude of synthesized acceleration is mainly liner model that could be a matter of offset since the dynamic movement is not regular. The approach used, is an acceleration decomposition which is a $3 D$ method. Since the three-axis accelerometer features are in some arbitrary orientation on the wearer's body ${ }^{[16]}$; therefore, the algorithm works as explained: a period of sampling interval is chosen to estimate a vertical acceleration vector on each axis corresponding to gravity component and then derived from the averages of all axis on the same sampling interval.

$$
A V=(A V x, A V y, A V z)
$$

Where $A V_{x}, A V_{y}$ and $A V_{z}$ are average vertical of each axis.

Also, the processing data is measured and hold the vector of acceleration on each axis at a given point in the same sampling interval represented as in equation 5.

$$
V=(V x, V y, V z)
$$

Where $\mathrm{V}_{\mathrm{x}}, \mathrm{V}_{\mathrm{y}}$ and $\mathrm{V}_{\mathrm{z}}$ are the vectors that made on the three acceleration at a given point of the period interval.

The vector $V$ in fact represents the acceleration that is caused by the gravity and the detail will represent in the result section. Therefore, the dynamic component of each axis that caused by the user's motion rather than gravity is can be expressed by formula 6 .

$$
d=(V x-A V x, A y-A V y, A z-A V z)
$$

Furthermore; in order to perform additional processing, the projection $p$ or the vector dot products of the dynamic component $d$ upon the average vertical axis $A V$ can be computed as in equation 7 , and that will help to deduce horizontal component of the dynamic acceleration $H$. 


$$
p=\left(\frac{d * A V}{A V * A V}\right) * A V
$$

The horizontal component of the dynamic acceleration $H$ can be expressed as 8 .

$$
H=d-p
$$

As a consequence, the result of the algorithm performs across a period of sampling interval estimates the magnitude of vertical components and the horizontal components of the dynamic accelerations, each of which is independent of the orientation of the object that wear the OEPS. The processed data of the accelerometer motions are then mapped to the contribution of the motion artefact that corrupted the desired signal and extraction of the reduced artefact PPG waveform that could be processed further to measure physiological information such as heart rate, oxygen saturation, heart rate variability and respiration rate.

\section{RESULTS}

The conceptual prototype behind implementing the automatic cancellation technique realizes by using Matlab (The MathWorks Inc., USA), ISIS professional software (Labcenter Electronics Ltd, UK) simulation and a test bench breadboard laboratory. In addition, a LabView (National Instruments Co., USA) design was used in both simulation and real experiment setups.

\subsection{Simulation procedures}

Figure 6 shows the Matlab simulation which was employed to recover the desired signal out of the captured one. The simulation used sinusoidal signals that were assimilated as real signals. For example, the first signal (signal $A$ ) represents the PPG signal and the second one (signal $B$ ) is a noise. The third signal (signal $C$ ) results from added previous signals, whereas signal $D$ is the precise signal of the first one (signal $A$ ) after applying the differential technique.

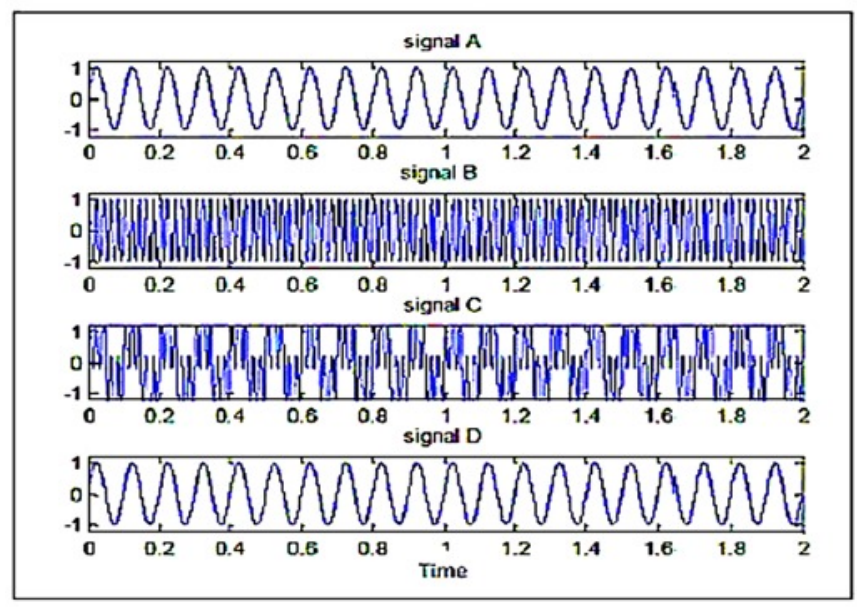

Figure 6. Differential technique to recover the original signal.

The technique design was also tested with the PPG signal instead of the sine wave. Figure 7a and b shows the circuit that implemented in ISIS professional to verify the success of this technique. The testing circuit includes the corrupted PPG signal buried in different levels of noise. 


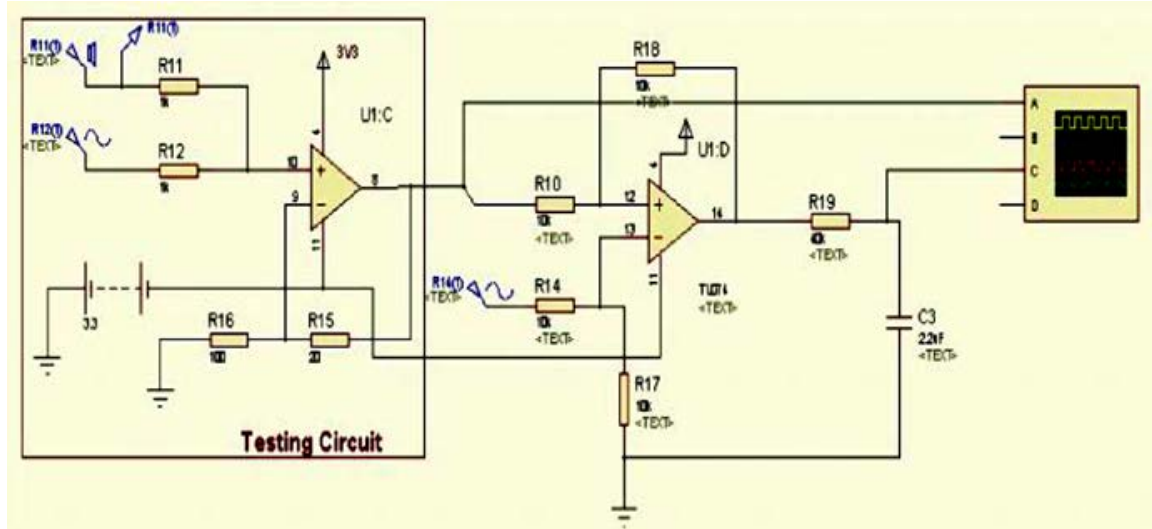

Figure 7.a. Differential function technique to detect the PPG signal.

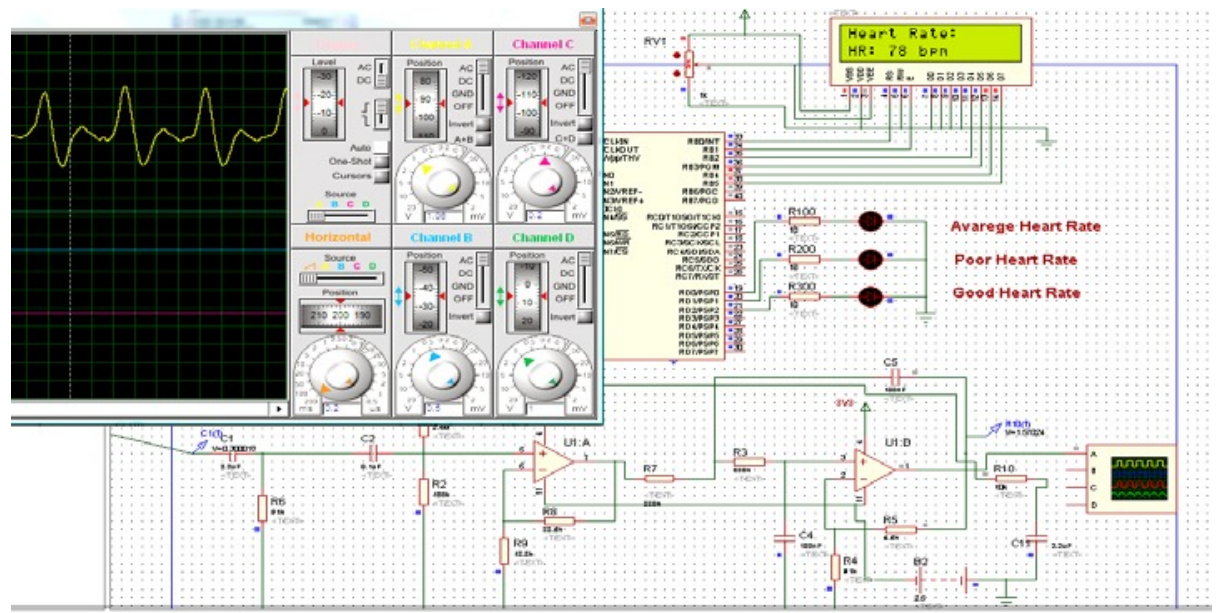

Figure 7.b. Detection the PPG signal and display heart rate on the LCD.

The output of the testing circuit represents the corrupted PPG produced from the movement as motion artefact. The reference signal was used as a signal as would be generated from the accelerometer. Figure 8 illustrates the output of the system design that has been implemented in the simulation. The PPG signal recovers which has been buried in the noise by using filters and differential technique.

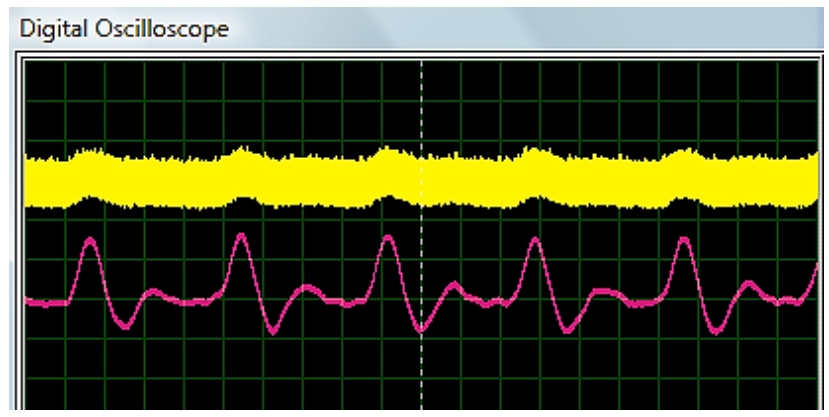

Figure 8. The extraction of the PPG signal after applying differential technique.

Furthermore; the ISIS professional software is being interfaced with the LabView design through a serial com port RS232 in order to verify the fundamental of the system design as well as to test the LabView GUI design. Figure 9 shows the receiving $P P G$ signal in the LabView GUI. 


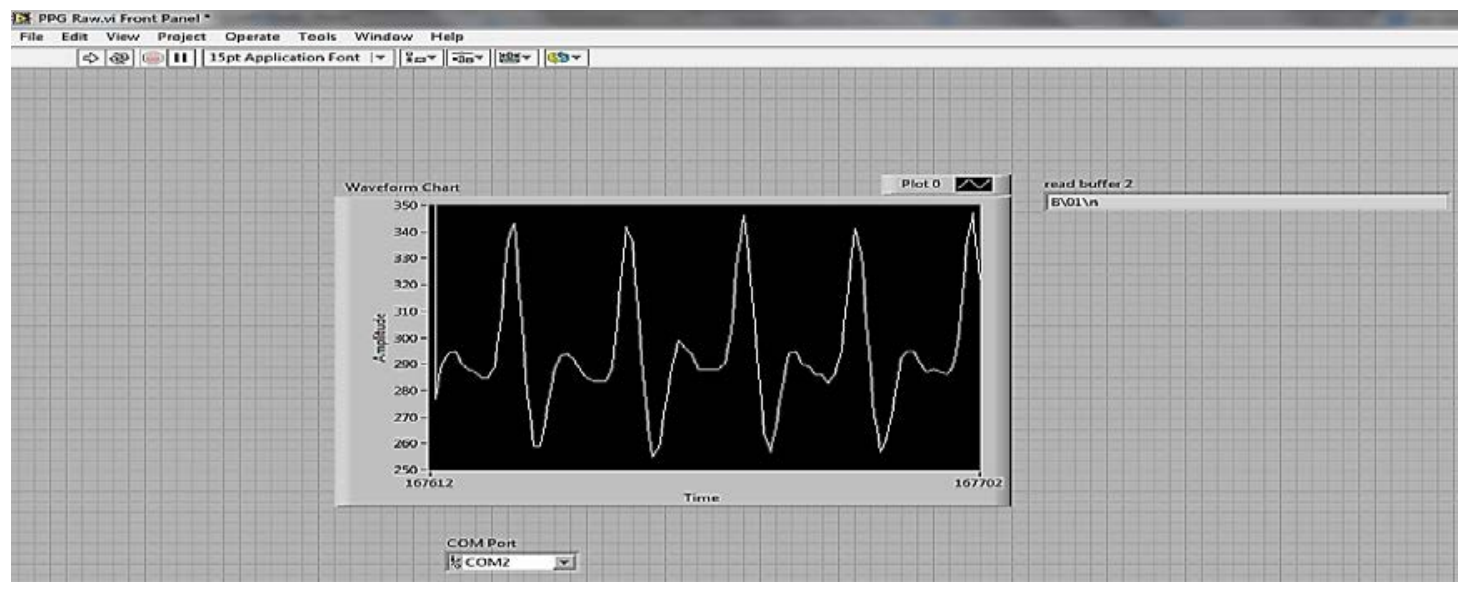

Figure 9. Receiving PPG through serial com port VISA

\subsection{Experimental results with the opto- electronic patch sensor OEPS hardware setup}

The design also implemented in the lab was initially assembled on a breadboard, to facilitate adjustment and choose the appropriate values of electronic components. The prototype was implemented in different ways and methods to make sure that the system design works properly. First and foremost, the PPG signal was tested through an oscilloscope with different optical sensor. The following figures show the waveforms of the obtained signal of the analogue front end. Figure $14 \mathrm{a}$ and $\mathrm{b}$ show a test measurement that presents a clear $P P G$ waveform signal with frequency of $1.4 \mathrm{~Hz}$ and amplitude of $1.3 \mathrm{~V}$ respectively. This test was measured in a static and relaxing mode, where there is no movement or physical activity during the examination. The scale measurement for both frequency and amplitude can be calculated as shown in Figure 14a and 14b. The time period of the signal is found around 2.8 multiply by the scaling factor of $250 \mathrm{~ms}$ and that result on approximately 0.7 which means that the frequency of the signal is $1.4 \mathrm{~Hz}$ as shown in Figure $10 \mathrm{a}$. Also, in Figure $10 \mathrm{~b}$ the amplitude is $1.32 \mathrm{~V}$ with scaling factor of $1 \mathrm{~V}$.

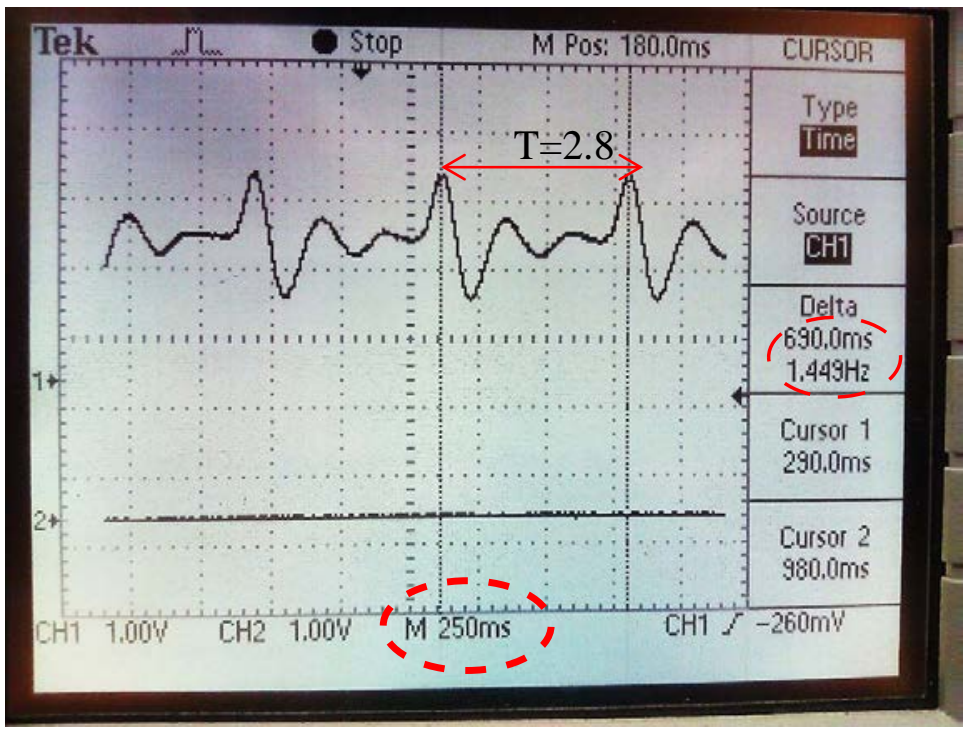

Figure 10.a. Detection of the PPG signal with frequency $1.4 \mathrm{~Hz}$. 


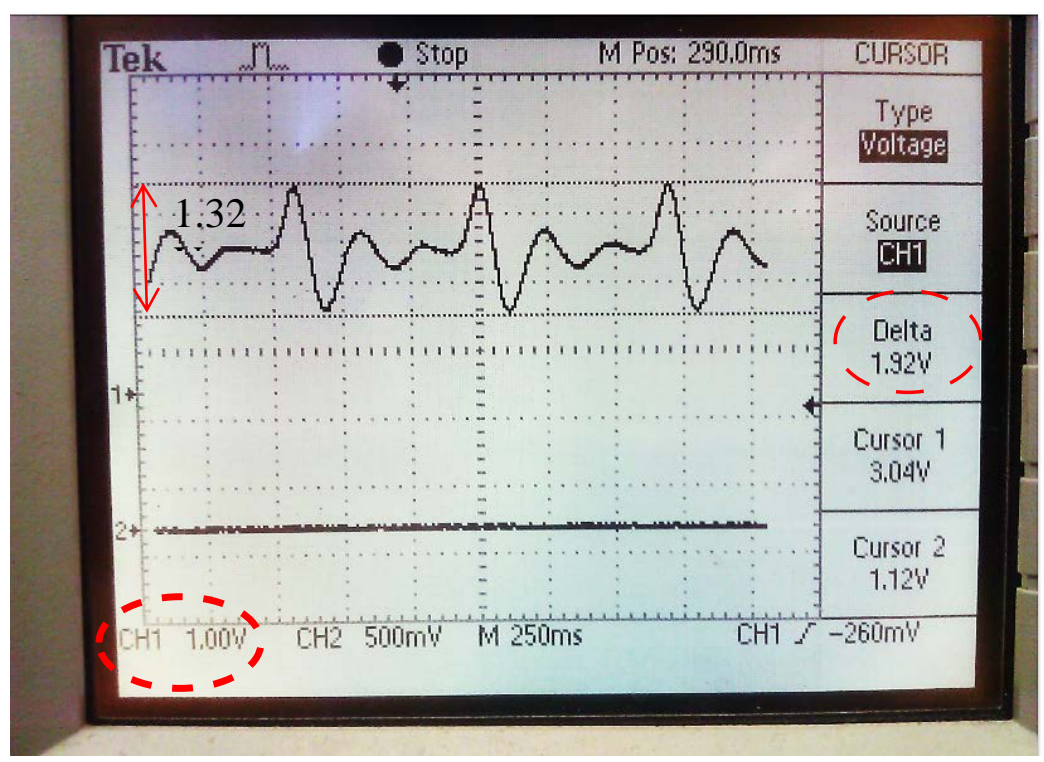

Figure 10.b. Detection of the PPG signal with amplitude 1.32V.

In addition, the reflection probe (Nellcor ${ }^{\mathrm{TM}}$ Max-Fast $^{\mathrm{TM}}$, COVIDIEN) was used with the scaling of 500ms as shown in Figure 11. However, there is still some noise that can be removed with further processing.

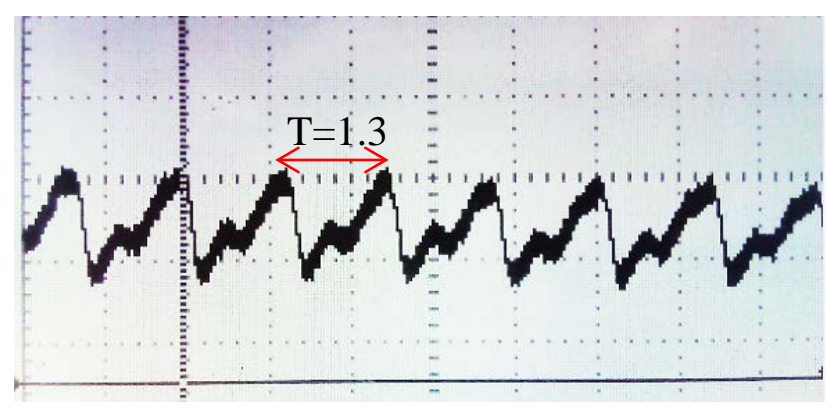

Figure 11. Reflectance mode detection of PPG signal.

Moreover, the opto-electronic patch sensor OEPS was tested which produces a clear signal as shown in Figure 12.

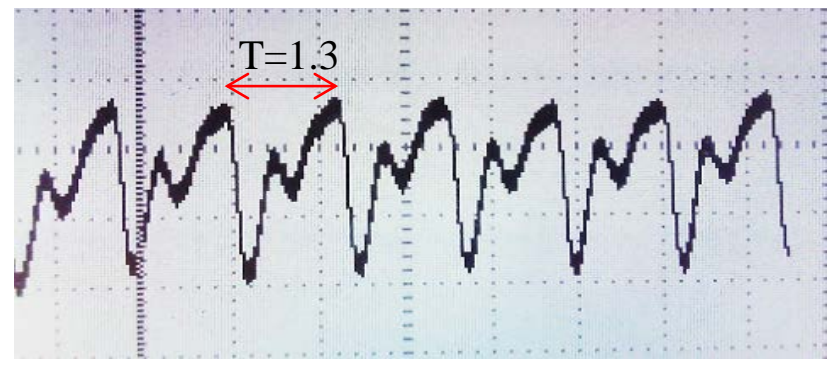

Figure 12. The OEPS detection of PPG signal

Furthermore, both the OEPS and a commercial reflectance contact pulse oximetry probe (Nellcor ${ }^{\mathrm{TM}}$ Max-Fast $^{\mathrm{TM}}$, COVIDIEN) were placed on the participant's finger. The PPG signals from two probes were captured simultaneously by a 4-channel PPG board (DISCO4, Dialog Devices Ltd., UK). The analogue-to-digital conversion for these captured PPG signals was implemented by a data acquisition board (USB-6009, National Instruments Co., USA), and the control software of the PPG board was performed by LabView GUI (National Instruments Co., USA). The desire signal is measured through the serial port RS232 on the PC and displays the PPG signals on the waveform. Figure 13 shows two screens shot that have been measured on the same volunteer at the same time with two different fingers. 


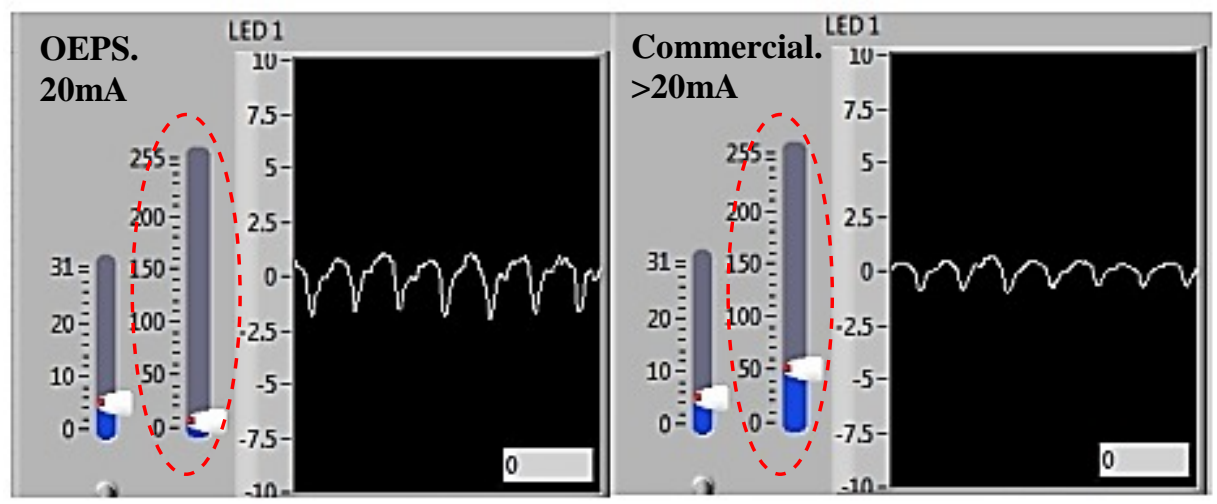

Figure 13. Detection the PPG, in left side with OEPS and the right one by commercial probe.

Also, Figure 14 represents the PPG signal that has taken from OEPS.

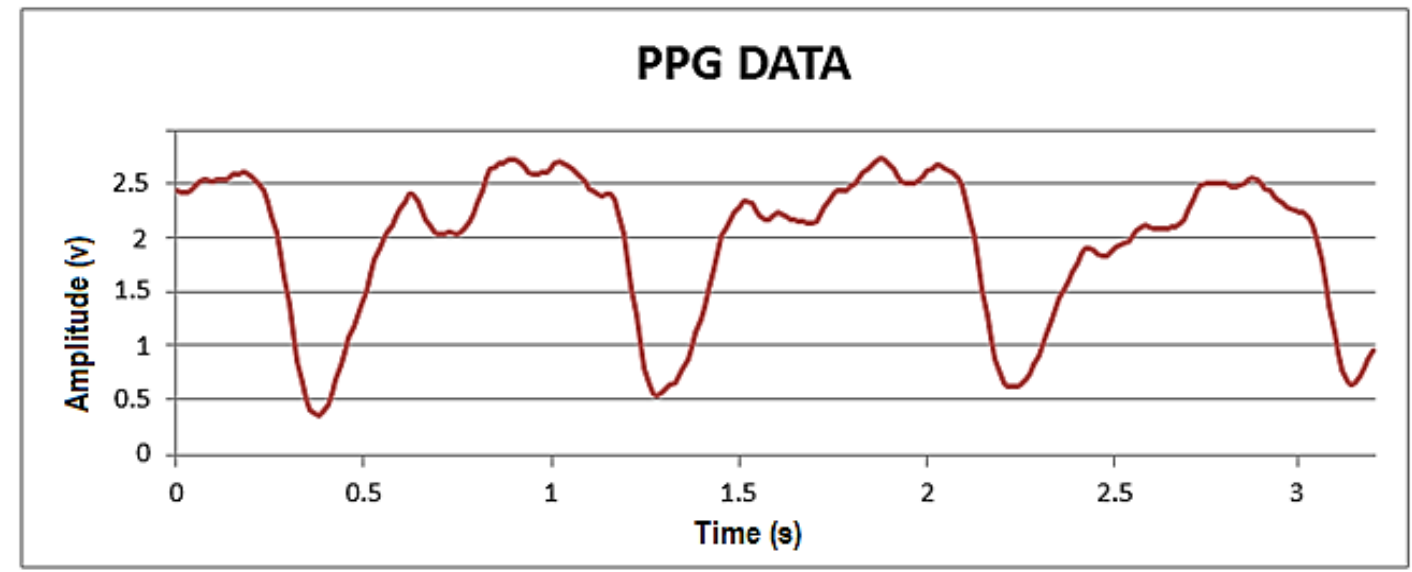

Figure 14. Detection the PPG with OEPS.

It is also noted that the OEPS was designed in this study consumed less power than the commercial sensor since the patch uses less current gain as shown in a dash circle in Figure 13. The analysis data confirmed that the OEPS has minimal and lower power consumption compared to that consumed from the commercial sensor as shown in Figure 15.

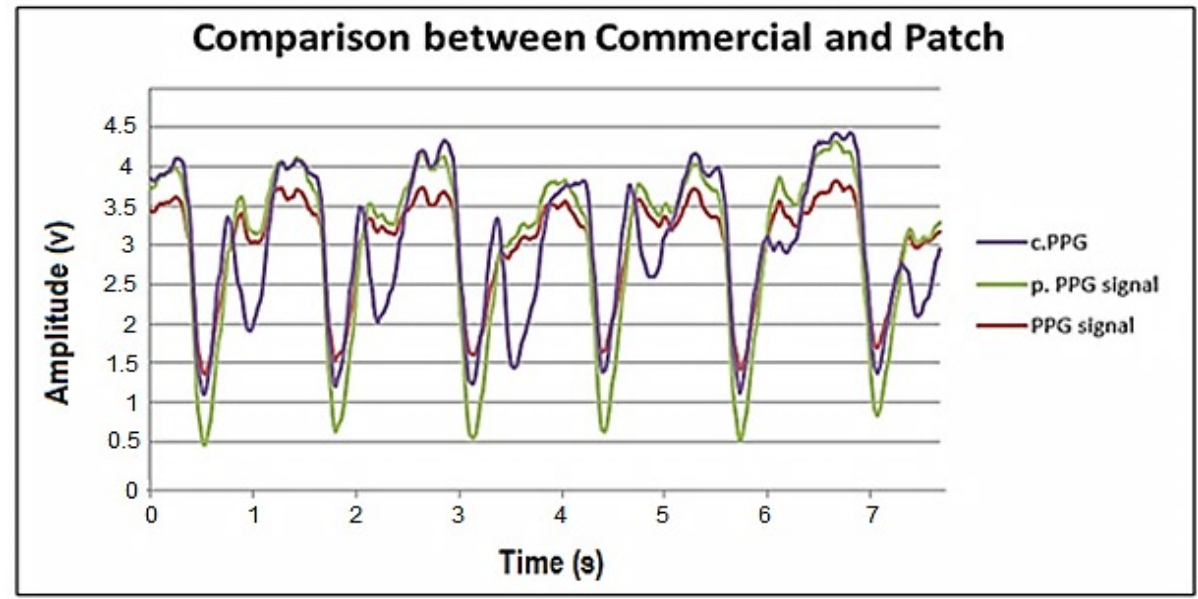

Figure 15. Signals comparison of power consumption, $\mathrm{p}=$ patch and $\mathrm{c}=\mathrm{commercial}$ 
Figure 15 shows clearly that the signal measured out of the OEPS had the same amplitude with the signal captured by a commercial sensor, even though; the OEPS operates with low gain current as shown in Figure 13. In addition, the trough of the patch's signal is bigger than the commercial sensor's signals. One important finding was that the OEPS has highly motion resistance than the commercial sensor due to four channels which present in this OEPS as can be seen in Figure 16.

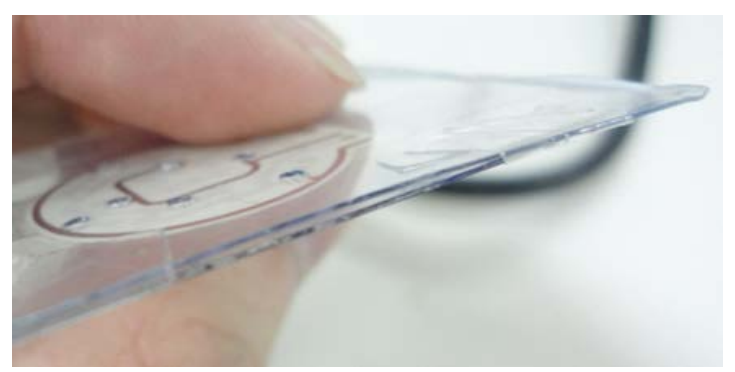

Figure 16. Flexible opto-electronic patch sensor (OEPS).

Figure 17 shows that the comparison of resistivity movement of the OEPS and the commercial sensor. It can be seen that the OEPS is more resistive than the commercial one and the signals still have their features which can be easily extracted to derive the critical information.

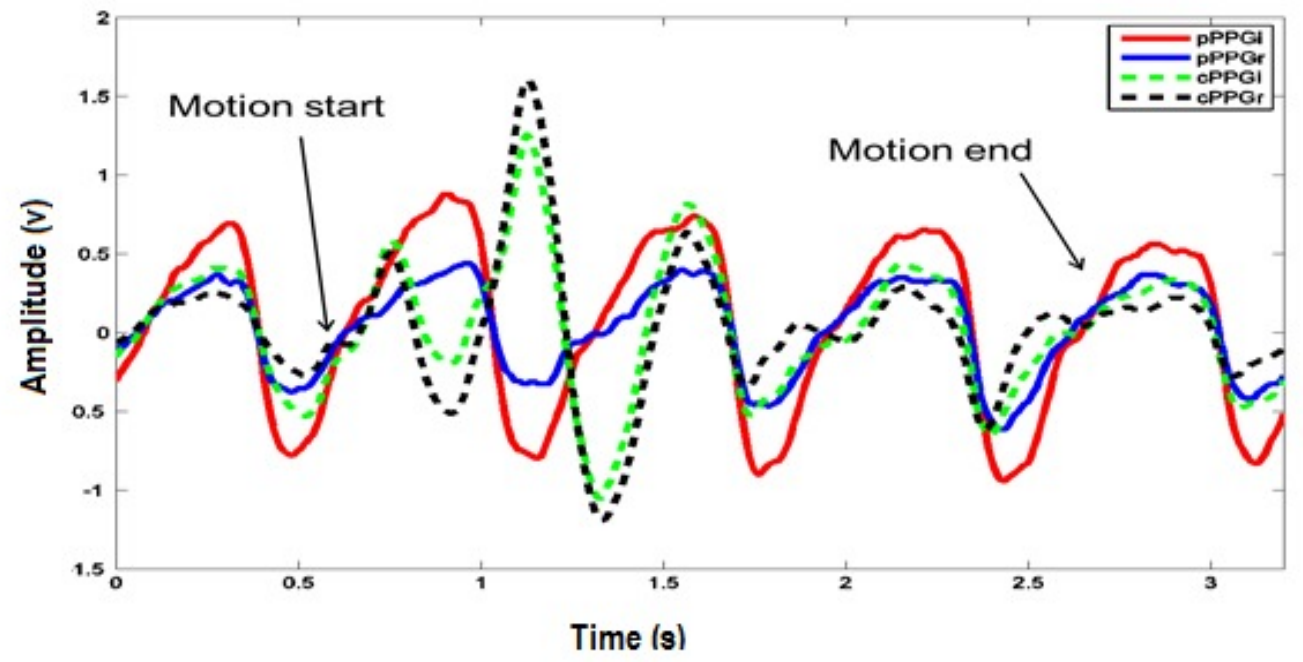

Figure 17. Signals comparison when motion occurred. $\mathrm{i}=$ infrared LED channel, $r=r e d$ LED channel, $\mathrm{p}=$ patch and $\mathrm{c}=$ commercial

However, in the presence of intensive movement, the PPG waveform corrupted and sometimes it is difficult to extract the physiological information through the corrupted signal. As a result in this study, the tri axis accelerometer, $A D X L 337$ presents the ability to extract the reduce noise PPG signal. The accelerometer was tested by the oscilloscope and voltmeter. Each measurement of accelerometer senses along with $X$-axis, $Y$-axis and $Z$-axis. For example, readings of $(x$, $y, z$ ) from the accelerometer have been taken in three different positions when there was not any movement. The first measurement was taken when the accelerometer sitting flat on a table, the gravitational acceleration is $0 \mathrm{~g}$ on $x$ and $y$-axis. The second measurement was taken when the accelerometer is placed $90^{\circ}$ vertical to the table. The third state is that when the accelerometer placed $-90^{\circ}$ which represent the reverse vertical to the table and that corresponding to $-1 \mathrm{~g}$ on the $z$ axis. Figure 18 shows the output response of the acceleration verses gravity. 

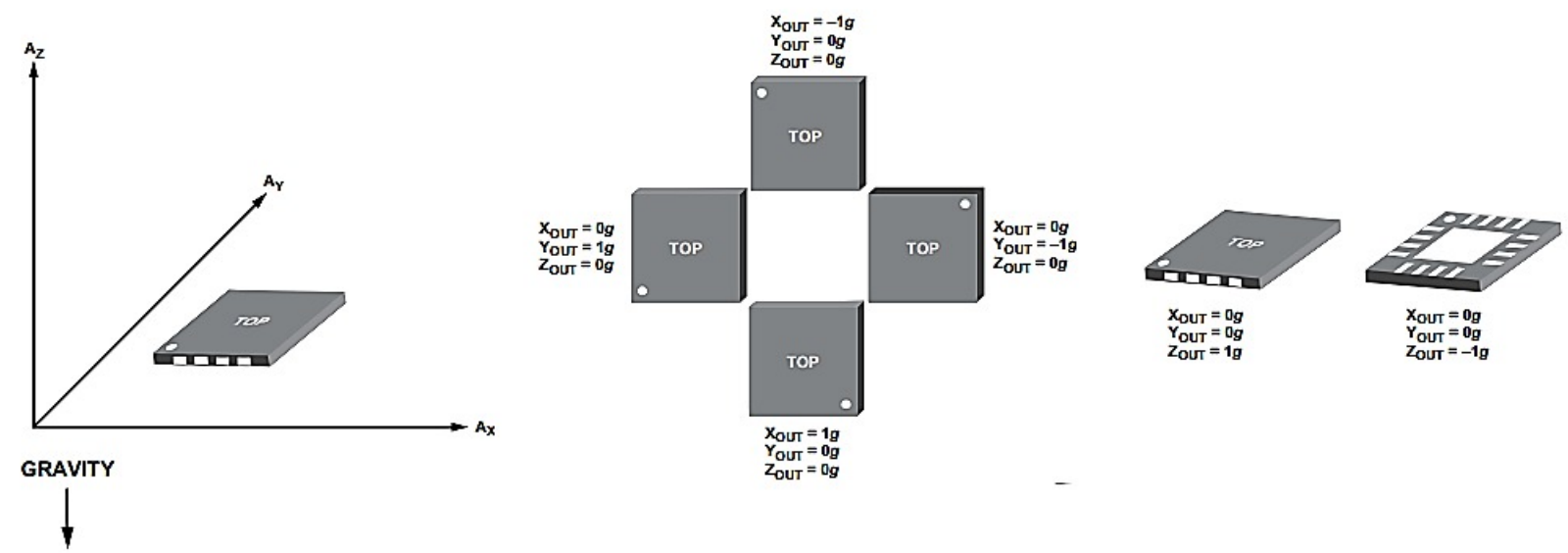

Figure 18. Output Response vs. Orientation to Gravity ${ }^{[17]}$

All three calibrations have been taken according to the gravity and table 1 summaries the measurements of different axis's state.

\begin{tabular}{|l|c|c|c|c|}
\hline State & Calibration method & Vx (v) & Vy (v) & Vz (v) \\
\hline Acc. On the table & oscilloscope & 1.80 & 1.60 & 1.60 \\
\cline { 2 - 5 } & voltmeter & 1.73 & 1.48 & 1.48 \\
\hline Acc. is vertical & oscilloscope & 1.40 & 1.85 & 1.85 \\
\cline { 2 - 5 } & voltmeter & 1.40 & 1.62 & 1.62 \\
\hline $\begin{array}{l}\text { Acc. is reverse } \\
\text { vertical }\end{array}$ & oscilloscope & 1.45 & 1.42 & 1.42 \\
\cline { 2 - 5 } & voltmeter & 1.45 & 1.30 & 1.30 \\
\hline
\end{tabular}

Table 1: Calibration of accelerometer offset along with 3 axes.

This observes that the calibration measurements are close to the offset reading in the datasheet of the accelerometer and it is important to compensate the processing data in the system design. Also, the measurement has taken along with physical movement. It shows that the acceleration signal along with three axis $\mathrm{x}, \mathrm{y}$ and $\mathrm{z}$ and the corrupted PPG signal. Figure 19 shows the derived PPG signal whiles the participant was moving, stopped and again moving. It can be clearly seen that if the wearer was doing any kind of activity the PPG signal is started to corrupt and in the rest duration the PPG will be seen obviously as marked in the figure. 


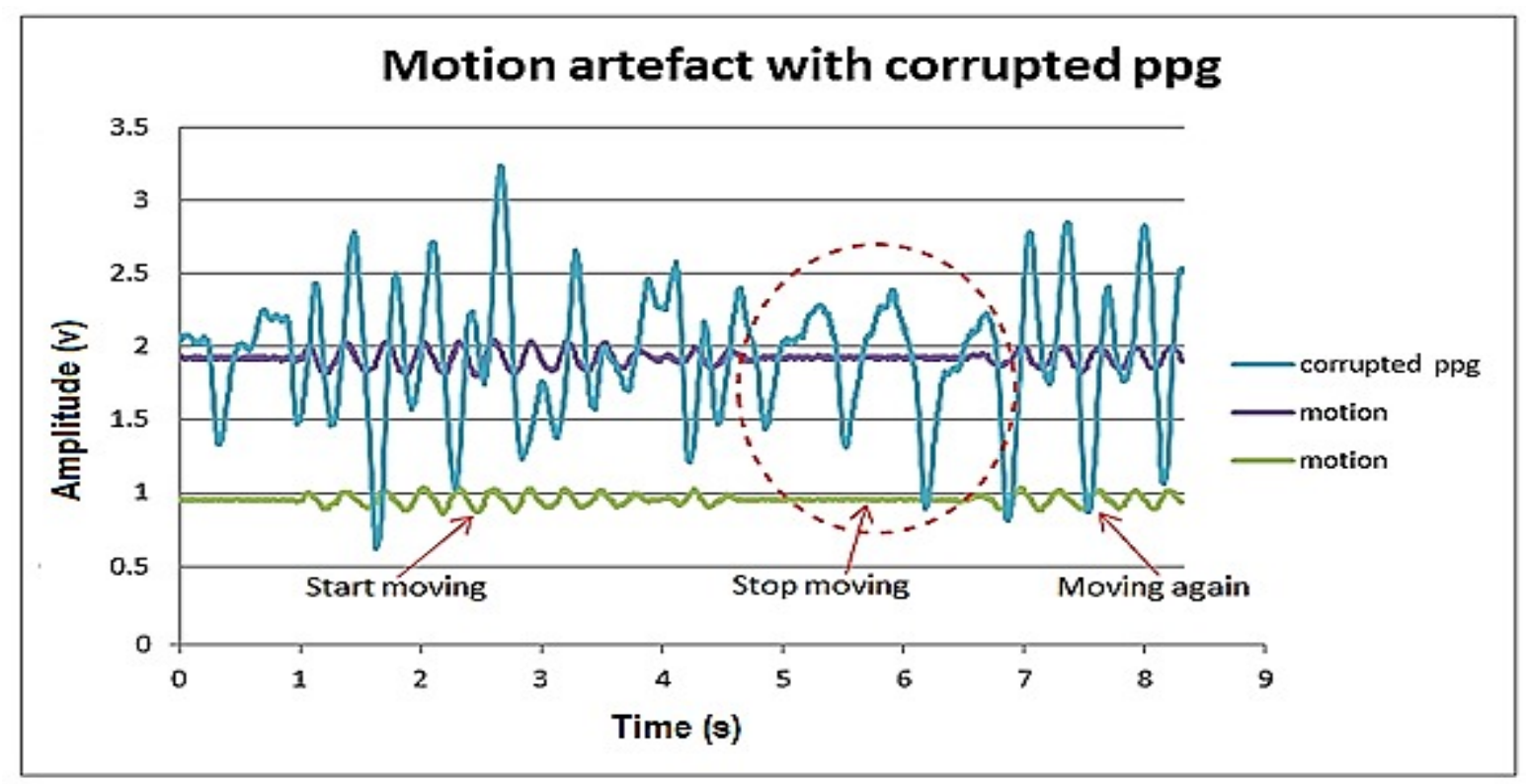

Figure 19. The corrupted PPG signal with movement.

The similar experiments were also implemented and the results displayed on the Oscilloscope with scaling of 500ms are presented in Figure 20.

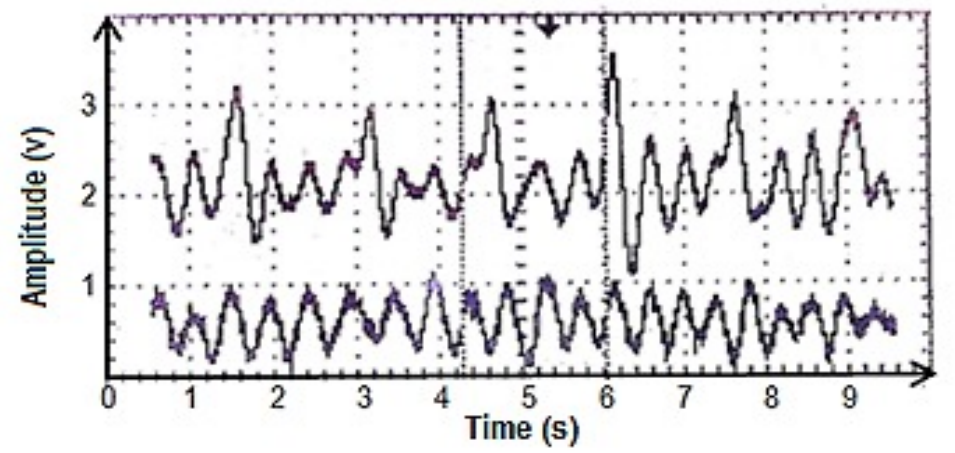

Figure 20. The corrupted PPG signal (above) with the output of the accelerometer (bottom).

Figure 21 shows the meeting between the two signals of accelerometer and corrupted PPG and the synchronization between them.

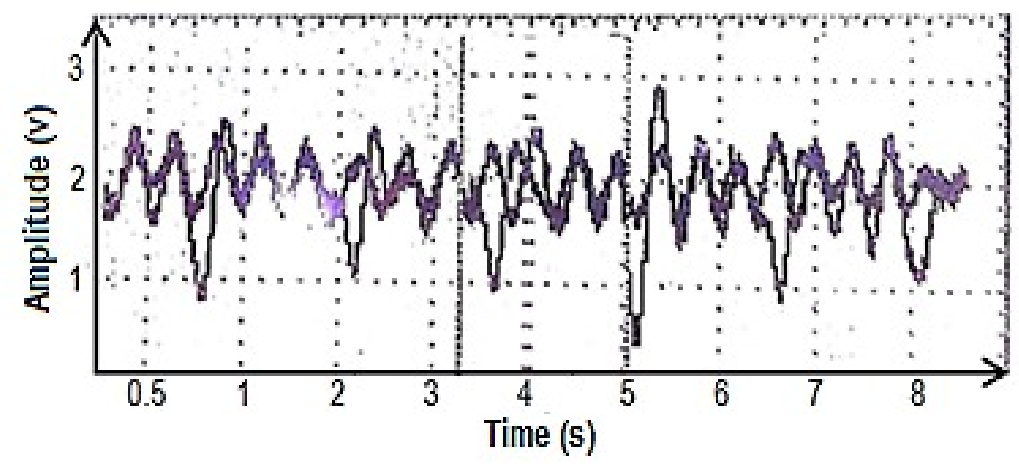

Figure 21. The corrupted PPG signal with the output of the accelerometer. 
In the final stage, the PPG signal with the differential artefact motion cancellation can be recovered, and the results as shown in Figure 22.

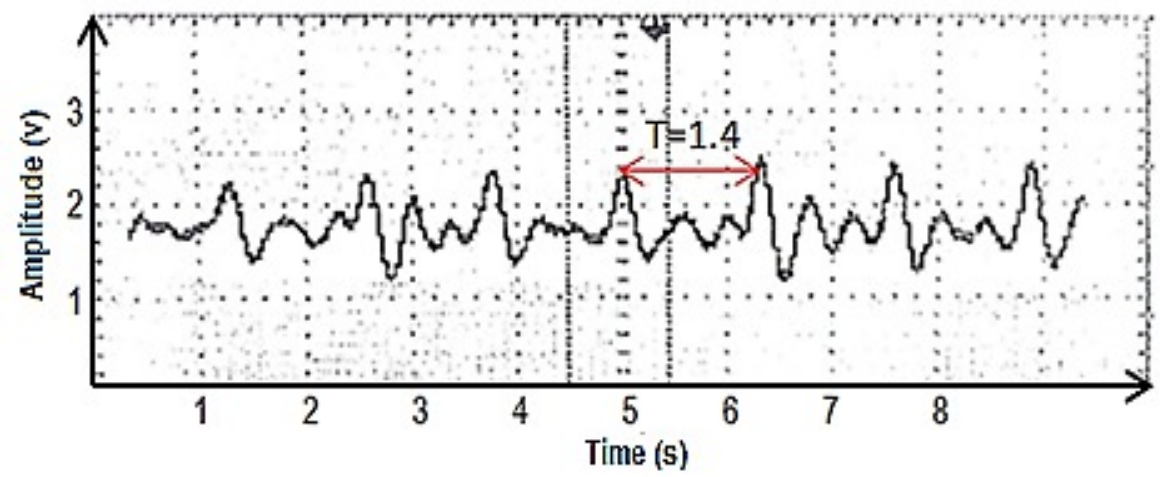

Figure 22. The PPG signal after applying differential artefact motion cancellation techniques.

One aim in this study was to send the human critical physiological parameters in the real time through a wireless network and that was achieved by using Bluetooth module $R N-42$ (Roving Network Co.). Figure 23 shows the PPG signal is captured and continuous monitoring as a real time on the LabView GUI.

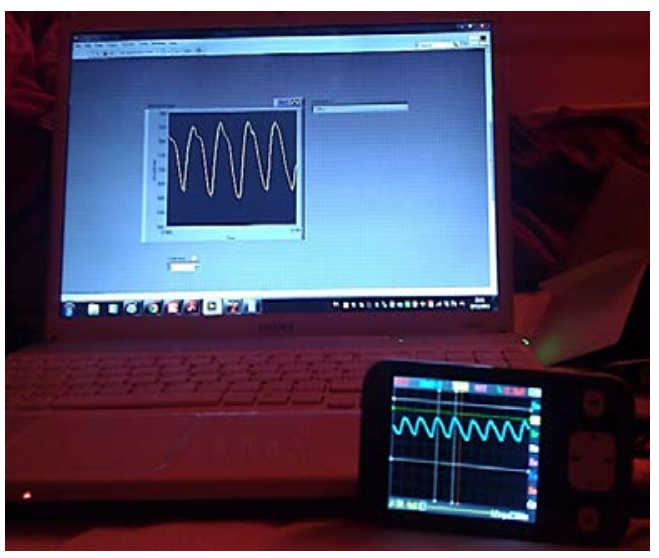

Figure 23. Synchronization of the PPG signals in a real time monitoring through a Bluetooth

Figure 24 shows the initial design that includes the optical sensor along with electronics and processing unite.

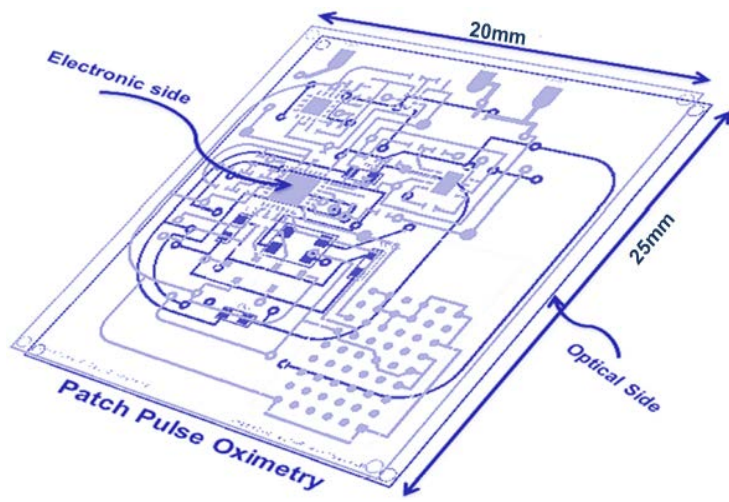

Figure 24. Initial design $3 D$ with optical side in the bottom and electronics in the top. 


\section{DISCUSSION}

The PPG signals derived from OEPS can be treated in the same way as any conventional reflectance PPG sensor. Two components from the PPG signals: a $D C$ component which is a relatively constant value or as a quasi-DC component that depends on the non-pulsatile tissues (skin, venous, etc.) and breathing cycle, and an $A C$ or pulsatile component which is synchronous with heart rate $(H R)$, and in turn can be used to extract a critical information such as heart rate, oxygen saturation, blood pressure and heart rate variability which allows to access the nervous system through sympathetic and parasympathetic mode.

However, the $A C$ component, is sensitive and easily corrupted by any physical movement. In order to recover and obtain the meaningful PPG signals, this study has researched solutions that overcome these problems and verifying these through simulation and hardware implementation in different physical activate scenarios. The first attempt was to use the simulation of various sinusoidal signals to assess and evaluate the performance of the design as shown in Figure 6. Realistically, the sinusoidal signal in the present consideration could be arguable as the OEPS system cannot depend on the performance of sinusoidal signal. Since the reality of the OEPS is much more complex than in the simulation and the physiological signals are highly subjective to the noises adopt in the measurement. The similar design for the OEPS was tested with the corrupted PPG signal instead of an ideal sinusoidal signal. Hence, the clear PPG signal could be extracted as shown in the results depicted in Figure 8. The simulation results indicate that the concept of OEPS system design is sound with motion artefact elimination methods tested in simulation.

In the practical environment, measurements were taken to prove the expectation of motion reduction in either relaxing or moving states. Even the experimental results present that in the case of activity absence, the PPG signal was easily detected as shown in Fig 10,11and 12 . In the situation of mechanical movement, the waveform of PPG was corrupted as shown in Figure 19. The results in Figure 21 demonstrate that there is a strong correlation of the corrupted PPG signals and the reference signals taken by the accelerometer simultaneously. Such a correlation of the corrupted signals and the reference signals is a great interesting how to work out an effective way in overcoming move artefacts. Figure 22 presents the $P P G$ signals with less noise after applying the algorithm in the processor platform as an alternative automatic artefact motion reduction technique. Even though, there is still some small portion of unwanted noise to be considered in the future work in order to reach a higher standard of OEPS with a robust design.

The proposed system together the body temperature sensor with the 3-axis accelerometer could provide a powerful bioinformatics platform capable of accurately detecting critical human physiological signs, i.e. heart rate, SaO2\%, respiration, body temperature, blood pressure, physical movements, even heart rate variability. In addition, from the data in Figure 15, it is apparent that the OEPS consumed less power than the commercial sensor since the gain current of the OEPS is smaller than that used for the commercial sensor as appear in Figure 13. Surprisingly, the OEPS that was designed in this study was found to be more resistive/resilient than the commercial sensor as shown in Figure 17 because the OEPS uses four channels to illuminate the skin. During the rest or static mode, both sensors work properly and can easily detect the PPG signal; however; in the presence of movement the desired signal will be corrupted and the OEPS shows less effected. However, more research work on this topic needs to be undertaken to more clearly understand the impact of physical activities on physiological phenomenon.

\section{CONCLUSION}

PPG centred human vital signs measurement together with body temperature sensors and 3-axis accelerometer is a new avenue for non-invasive and wearable biomedical monitoring techniques for arterial pulsations in vivo. In the present research work, a novel yet cost-effective physiological measurement has been successfully demonstrated as a viable concept for continuous monitoring of real-time wireless personal healthcare for ageing population and even individual family members.

The design of motion artefact cancellation methods based on a reference signal of the accelerometer is proofed with a good performance of physiological measurement as described. The technical method of a system design depends on the fact that the primary input of the corrupted signal is synchronized with the reference signal captured simultaneously from the 3 axis accelerometer.

This approach is capable of acquire and process physiological signals precisely and in real time. Finally, the preliminary results and current findings add substantially to our understanding of interaction on illumination to the human tissue and correlation of the pulsatile waveforms with contributory noise effects on physiological measurements. The results are 
encouraging and will lead the assembly of a practical patch sensor towards a prospectus of low-cost homecare tool. Clinically this is also a useful device as a cost-effective means to overcome motion artefacts, size, real time, secure wireless communication and poor signal-to-noise ratio.

\section{ACKNOWLEDGEMENT}

The authors would like to express their thanks to Loughborough University and The Ministry of Higher Education in the Kingdom of Saudi Arabia represented at Taif University for this study and the financial support to carry out this study. Also, the authors would like to acknowledge the $7^{\text {th }}$ European Community Framework Program for financial support through a Marie Curie International Research Staff Exchange Scheme (IRSES) Project entitled "Micro-Multi-Material Manufacture to Enable Multifunctional Miniaturised Devices (M6)” (Grant No. PIRSES-GA-2010-269113).

\section{REFERENCES}

[1] Australian Institute of Health and Welfare, "Heart, Stroke and Vascular Disease-Australian Facts 2004," Canberra National Heart Foundation of Australia (2004).

[2] Good health adds life to years: Global brief for World Health Day 2012, WHO (2012).

[3] Hu S, Azorin-Peris V, Zheng J, "Opto-physiological modeling applied to photoplethysmographic cardiovascular assessment," J Healthc Eng, 4(4) 505-28 (2009).

[4] Koelwyn GJ, Wong LE, Kennedy MD, Eves ND, "The effect of hypoxia and exercise on heart rate variability, immune response, and orthostatic stress ," Scand J Med Sci Sports, 23(1) e1-8 (2013).

[5] Lopez, S, "Pulse Oximeter Fundamentals and Design," Free- scale Semiconductor, Inc., application note document No AN4327 Rev., 1 (2011).

[6] Li Y, "Pulse Oximetry". Department of Electronic Engineering, University of Surrey, UK, SURJ 2,10 (2007).

[7] Coyle S, Morris D, Lau K T, Diamond D, Moyna N Coyle, "Textile-Based Wearable Sensors for Assisting Sports Performance ," Body Sensor Networks, IEEE Computer Society, 307-11 (2009).

[8] Curone D, Secco EL, Tognetti A, Loriga G, Dudnik G, Risatti M, Whyte R, Bonfiglio A, Magenes G, "Smart garments for emergency operators: the ProeTEX project, "IEEE Trans Inf Technol Biomed, 14(3) 694-701 (2010).

[9] Zysset C, Nasseri N, Büthe L, Münzenrieder N, Kinkeldei T, Petti L, Kleiser S, Salvatore GA, Wolf M, Tröster G, "Textile integrated sensors and actuators for near-infrared spectroscopy ," Opt Express, 21(3) 3213-24 (2013).

[10] Chan M, Estève D, Fourniols JY, Escriba C, Campo E, "Smart wearable systems: current status and future challenges," Artif Intell Med, 56(3) 137-56 (2012).

[11] Dresher, R., "Wearable Forehead Pulse Oximetry: Minimization of Motion and Pressure Artifacts," Thesis submitted for the Degree of Master of Science Worcester, USA, Worcester Polytechnic Institute, Department of Biomedical Engineering (2006).

[12] Sun Y, Hu S, Azorin-Peris V, Greenwald S, Chambers J, Zhu Y, "Motion-compensated noncontact imaging photoplethysmography to monitor cardiorespiratory status during exercise," J Biomed Opt, 16(7), 077010 (2011).

[13] Domingues A., "Development of a Stand Development Stand- Alone Pulse Oximeter," Dissertation presented to the University of Coimbra to obtain the degree of Master of Biomedical Engineering (2009).

[14] Yeon, H., "Motion artifact reduction with active noise cancellation and accelerometer for wearable Photoplethysmography" Advanced Institute of Science and Technology, Biomedical Engineering Department, Daejeon, South Korea, Daejeon (2006).

[15] Bonnie G., "Quantitating Physical Activity in COPD Using a Triaxial Accelerometer," Chest, 117(5) 1359-67 (2000).

[16] Mizell D., "Using Gravity to Estimate Accelerometer Orientation", Proceedings of the Seventh IEEE International Symposium on Wearable Computers, (ISWC’03) 1530-0811/03 (2003).

[17] Analog Device , "Small, Low Power, 3-Axis \pm 3 g Accelerometer, ADXL337", Norwood, MA 02062-9106, USA, (2010) 